\title{
Zinc Ion-immobilized Magnetic Microspheres for Enrichment and Identification of Multi-phosphorylated Peptides by Mass Spectrometry
}

\author{
Se Won BAE, ${ }^{* 1}$ Jae Il KIM, ${ }^{* 2}$ Inseong ChOI, ${ }^{* 2}$ Jiha Sung, ${ }^{* 3}$ Jong-In Hong, ${ }^{* 4}$ and Woon-Seok YeO ${ }^{* 2 \dagger}$ \\ *1 Green Chemistry and Materials Group, Korea Institute of Industrial Technology (KITECH), Cheonan 31056, \\ Korea \\ *2 Department of Bioscience and Biotechnology, Bio/Molecular Informatics Center, Konkuk University, \\ Seoul 05029, Korea \\ *3 Department of Applied Chemistry, Dongduk Women's University, Seoul 02748, Korea \\ *4 Department of Chemistry, Seoul National University, Seoul 08826, Korea
}

\begin{abstract}
The selective isolation of phosphorylated peptides and subsequent analysis using mass spectrometry is important for understanding how protein kinase and phosphatase signals can precisely modulate the on/off states of signal transduction pathways. However, the isolation and detection of multi-phosphorylated peptides is still limited due to their distinct affinity to various materials and their poor ionization efficiency. Here, we report a highly efficient and selective enrichment of phosphorylated peptides using binuclear $\mathrm{Zn}^{2+}$-dipicolylamine complex-coated magnetic microspheres ( $\mathrm{ZnMMs}$ ). ZnMMs can utilize the rapid and selective isolation/enrichment of phosphorylated peptides and the subsequent mass spectrometric analysis, given the intrinsic magnetic property of magnetic microspheres and the highly selective binding ability of the binuclear $\mathrm{Zn}^{2+}$-dipicolylamine complex to phosphate groups. $\alpha$-Casein and $\beta$-casein were chosen for a proof-of-concept demonstration. We contemplated that phosphopeptides were selectively isolated and enriched from both the tryptic digests of casein proteins and mixed samples with a high degree of sensitivity by facilitating ZnMMs. Especially, ZnMMs showed high efficiency with multi-phosphopeptides, which are in general difficult to be examined by mass analysis on account of their poor ionization efficiency. For the model protein $\alpha, \beta$-casein mixture of the tryptic digest, 17 phosphopeptides were identified with ZnMMs and $82 \%$ of the enriched phosphopeptides were multiphosphorylated peptides, indicating that ZnMMs have excellent enrichment efficiency and strong affinity towards multiphosphorylated peptides.
\end{abstract}

Keywords Phosphopeptides enrichment, IMAC, mass spectrometry, molecular recognition, magnetic microspheres

(Received June 19, 2017; Accepted August 31, 2017; Published December 10, 2017)

\section{Introduction}

The addition and removal of phosphate groups to and from proteins by kinases and phosphatases, respectively, form the basis for the regulation of numerous cellular events, including cell growth, metabolism, and signal transduction. ${ }^{1-3}$ More than $30 \%$ of the proteins in the eukaryotic proteome are likely to be phosphorylated at some point during their existence. ${ }^{4}$ The most common phosphorylation event involves hydroxyl groups of three amino acids (i.e., serine, threonine, and tyrosine), although as many as six other amino acids have the potential for modification. Thus, the study of protein phosphorylation states is important for defining protein kinase substrates, and revealing the activation states of signal transduction pathways. ${ }^{5-7}$ Currently, high-throughput proteomic analysis is mainly accomplished by mass spectrometry (MS). ${ }^{8}$ However, the determination of phosphopeptides in complex biological

† To whom correspondence should be addressed.

E-mail:wsyeo@konkuk.ac.kr samples has remained a significant challenge because their scarcity in the total proteome as well as their deficient ionization and ion suppression during the mass spectrometric analysis. ${ }^{9-13}$ Furthermore, the analysis of multi-phosphorylated peptides is particularly more difficult than that of mono-phosphorylated peptides due to their even lower abundance and severe ion suppression in MS..$^{14,15}$ Therefore, the development of strategies for highly selective phosphopeptide enrichment is in great demand. ${ }^{16}$

Chelation/coordination chemistry is a most popular strategy among a number of approaches that have been developed for phosphopeptide enrichments. The key principle includes the use of immobilized metal ions (immobilized metal ion affinity chromatography, IMAC) or metal oxides (metal oxide affinity chromatography, MOAC) that can bind to peptide phosphate groups. ${ }^{17-19}$ The binding ability of immobilized cations in IMAC, such as $\mathrm{Fe}^{3+}, \mathrm{Ti}^{4+}$ and $\mathrm{Ga}^{3+}$, stems from the nature of metal cations, which are bound to chelating ligands on the surface of carrier resins such as iminodiacetic acids or nitrilotriacetic acids. ${ }^{20}$ Previous studies have demonstrated that IMAC shows a strong selectivity for multi-phosphorylated 


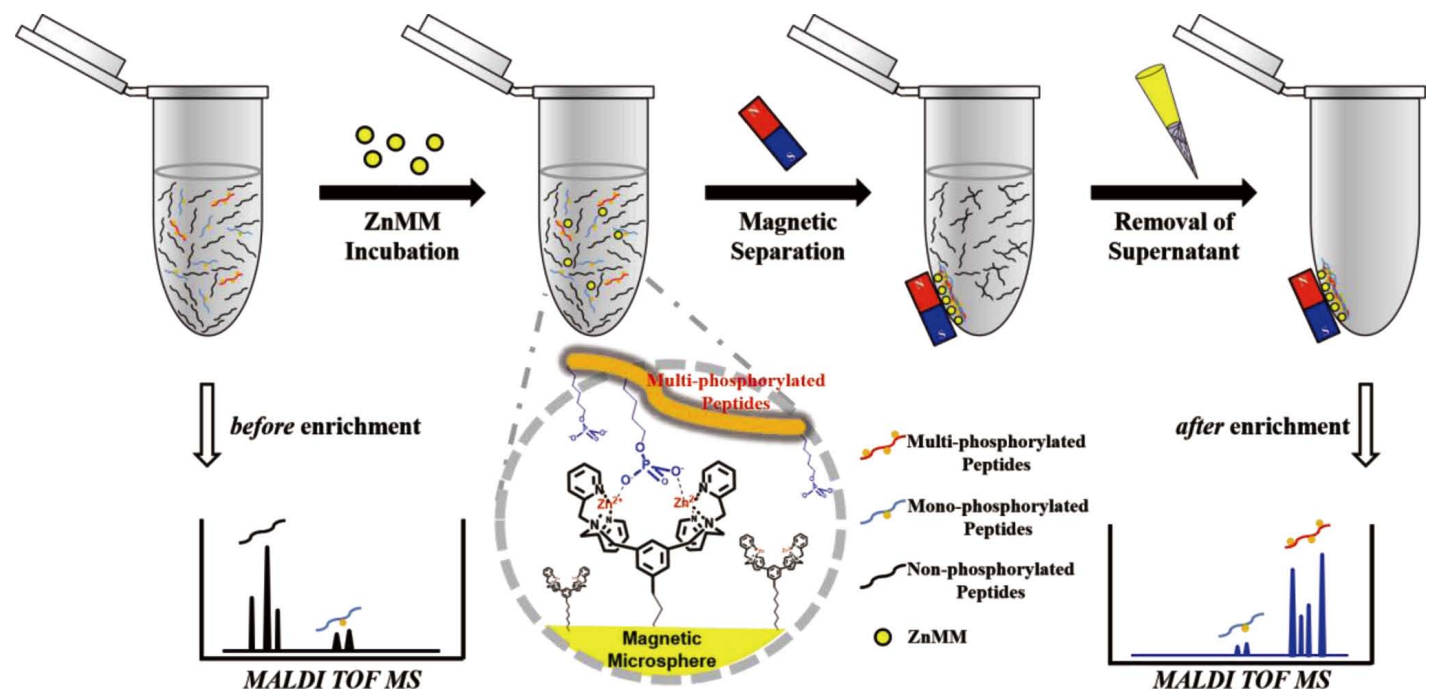

Fig. 1 The strategy of selective phosphopeptide enrichment using ZnMMs. The tryptic digest of proteins and the synthesized ZnMMs are incubated for $30 \mathrm{~min}$ at room temperature. Then, phosphopeptide-bound magnetic particles are separated by a magnet and the supernatant was removed. Finally, the enriched phosphopeptides are analyzed by MALDI-TOF MS.

peptides. ${ }^{11}$ However, the specificity of conventional IMAC materials is not ideal due to the non-selective co-enrichment of acidic peptides. ${ }^{21}$ MOAC also has been intensively applied for the selective enrichment of phosphopeptides based on Lewis acid-base interaction, using titanium, zirconium, aluminum, niobium, and other metal oxides. ${ }^{22-26}$ Recently, $\mathrm{TiO}_{2}$ has gained increasing attention and is regarded as one of the most powerful materials for phosphopeptide enrichment, particularly for isolating mono-phosphorylated peptides. ${ }^{27,28}$ Consequently, design and synthesis of new materials with high enrichment selectivity for multi-phosphorylated peptides are still demanded.

As such, we speculated that the introduction of a molecular moiety that specifically binds phosphate groups may overcome these shortcomings. Phosphate recognition via artificial receptors has risen over the past several decades. ${ }^{29,30} \mathrm{Zn}^{2+}$ dipicolylamine $(\mathrm{ZnDpa})$ is a commonly used artificial receptor that possesses two empty orbitals for forming stable zincoxygen complexes. In recent years, due to its high selectivity and stability, $\mathrm{ZnDpa}$ is regarded as a standard receptor for sensing and imaging of phosphate-containing biomolecules. ${ }^{31,32}$ In particular, the binuclear $\mathrm{ZnDpa}$ complex has been shown to coordinate phosphate groups with high affinity and selectivity. ${ }^{33-41}$ Here, we devised the binuclear $\mathrm{ZnDpa}$ complex-coated magnetic microspheres (ZnMMs) for the selective enrichment of phosphopeptides. In order to improve the selectivity of phosphopeptide enrichment and to take advantage of easy isolation from complex biological samples, we introduced two key modifications to the conventional IMAC adsorbent. First, magnetic microspheres that facilitate the enrichment strategy were chosen because of their excellent magnetic responsiveness, easy surface manipulation, and recovery. ${ }^{42-46}$ We utilized uniform, silica-based, $5 \mu \mathrm{m}$-sized superparamagnetic microspheres coated with high-density primary amine groups. ${ }^{47}$ These microspheres not only have excellent stability in strong acid and alkaline buffers, but also have a hydrophilic surface to minimize nonspecific adsorption. Second, the phosphate-selective artificial receptor, the binuclear $\mathrm{ZnDpa}$, was coupled, instead of iminodiacetic acids or nitrilotriacetic acids for the $\mathrm{Fe}^{3+} / \mathrm{Ga}^{3+}$ IMAC. This artificial receptor provides a suitable structural orientation for the binding of phosphate groups with very high specificity and affinity (the binding constant between binuclear $\mathrm{ZnDpa}$ receptor and phosphorylated peptides was reported as approximately $\left.10^{6} \mathrm{M}^{-1}\right) ;{ }^{38}$ therefore, the phosphopeptide enrichment is not hampered by highly acidic or multiple basic residues. Moreover, a large number of binuclear $\mathrm{ZnDpa}$ presented on the microsphere's surface can afford a preferential binding ability for multi-phosphorylated peptides. Figure 1 depicts the structure of ZnMMs and the phosphopeptide enrichment strategy for using ZnMMs. ZnMMs are mixed with a sample solution containing both phosphopeptides and nonphosphopeptides. After incubation, the phosphopeptide-bound magnetic particles are separated by external magnetic fields and subsequently analyzed by MALDI-TOF MS. ${ }^{48}$

\section{Experimental}

\section{Reagents and chemicals}

The magnetic particles were acquired from Bioclone Inc. (CA, USA). $\alpha$-Casein and $\beta$-casein were obtained from SigmaAldrich (MO, USA). BSA was acquired from SERVA Electrophoresis GmbH (Heidelberg, Germany). Trypsin was purchased from Promega (WI, USA). $\alpha$-Cyano-4-hydroxycinnamic acid (CHCA) was purchased from Bruker Daltonics (Leipzig, Germany).

\section{Synthesis of ZnMMs}

Amine group-functionalized magnetic microspheres $(1 \mathrm{mg}$, $250 \mu \mathrm{mol}$ of the amine functional group/g) were supplied to a solution of succinic anhydride ( $1 \mathrm{~mL}, 100 \mathrm{mM}$ in DMSO). The magnetic microspheres were cleansed with DMSO and the mixture was stirred gently at room temperature overnight. The resulting magnetic microspheres were immersed in a mixture of $N$-hydroxysuccinimide solution $(200 \mu \mathrm{L}, 5 \mathrm{mg} / \mathrm{mL}$ in DMSO) and 1-ethyl-3-(3-dimethylaminopropyl)carbodiimide (EDC, $200 \mu \mathrm{L}, 20 \mathrm{mg} / \mathrm{mL}$ in DMSO) for $3 \mathrm{~h}$. The magnetic microspheres were separated by a magnet and washed with DMSO to separate the NHS-activated magnetic microspheres. The binuclear 
(a)
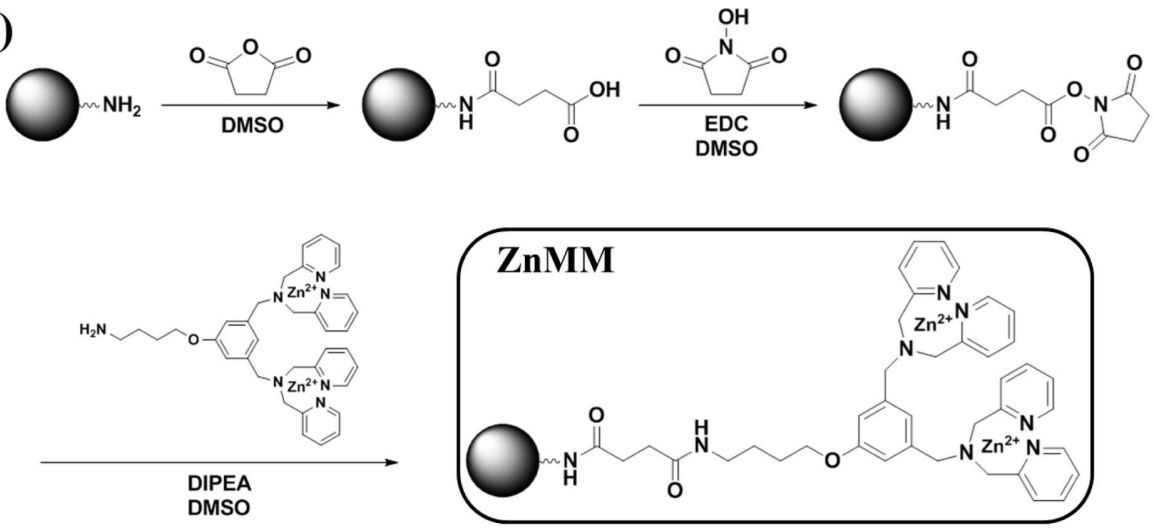

(b)

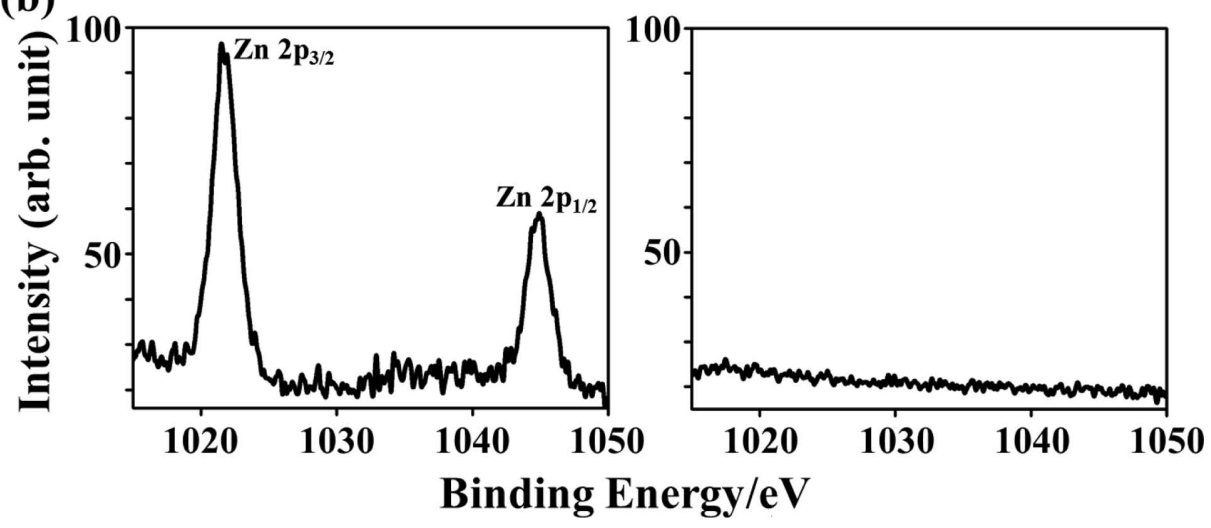

Fig. 2 (a) The synthetic procedure for the preparation of ZnMMs. (b) Zn 2p XPS spectra obtained from ZnMMs (left) and bare MMs (right).

ZnDpa complex $(200 \mu \mathrm{L}, 50 \mathrm{mM}$ in DMSO) was incubated with the NHS-activated magnetic microspheres in the presence of $50 \mu \mathrm{L} N, N$-diisopropylethylamine (DIPEA) overnight. The microspheres were washed with DMSO and separated by a magnet. The resulting $\mathrm{ZnMMs}$ were stored at $4^{\circ} \mathrm{C}$.

\section{XPS analysis of ZnMMs}

The ZnMMs and bare magnetic microspheres that were adsorbed on a silicon wafer $(0.5 \times 0.5 \mathrm{~cm})$ were analyzed by an ESCA 2000 (Thermo VG Science, USA) with a twin X-ray source $(\mathrm{Mg} / \mathrm{Al}$ target).

\section{Tryptic digestion of proteins}

The trypsin solution $(10 \mathrm{ng} / \mu \mathrm{L}$ in $2 \mathrm{mM}$ ammonium bicarbonate, $0.5 \mathrm{mM}$ calcium chloride, and $10 \%$ acetonitrile at $\mathrm{pH}$ 7) was mixed with proteins in a ratio of 1:100 (trypsin: protein $(\mathrm{w} / \mathrm{w}))$ and incubated at $37^{\circ} \mathrm{C}$ for $15 \mathrm{~h}$.

\section{Phosphopeptide enrichment using ZnMMs}

For the enrichment of the phosphopeptides from the $\alpha$-casein and $\beta$-casein digests, $100 \mu \mathrm{L}$ of the sample solution was mixed with $4 \mu \mathrm{L}$ of the ZnMMs $(1 \mu \mathrm{g} / \mu \mathrm{L})$ in HEPES buffer $(1 \mathrm{mM}$, $\mathrm{pH}$ 7.4) with gentle shaking for $30 \mathrm{~min}$. The supernatant was eliminated after a separation by a magnet and the microspheres were cleansed with water. MS analysis was performed directly without any releasing processes.

\section{MALDI-TOF MS analysis}

The mass analysis was performed using an Autoflex III MALDI-TOF mass spectrometer (Bruker Daltonics, Germany) equipped with a Smartbeam laser as the ionization source. The mass spectra of the positive/negative ions were acquired in the reflector mode under the following parameters: 19/-19 kV accelerating voltage, $50 \mathrm{~Hz}$ repetition rate, an average of $\sim 500$ shots, and CHCA $(1 \mathrm{mg} / 150 \mu \mathrm{L}$ in $50 \%$ acetonitrile, $0.1 \%$ TFA in distilled water) as a matrix.

\section{Results and Discussion}

ZnMMs were constructed via the immobilization of the binuclear ZnDpa complex on the amine-presenting microsphere's surface (Fig. 2a). The binuclear ZnDpa complex was synthesized with the modified method reported by Smith et al. (see Fig. S1 in Supporting Information). ${ }^{49} \mathrm{Dpa}$ is known to be strongly bound to $\mathrm{Zn}^{2+}$ with $\mathrm{K}_{\mathrm{a}}$ of approximately $10^{7} \mathrm{M}^{-1}{ }^{49}$ and therefore, $\mathrm{Zn}^{2+}$ was easily inserted to give $\mathrm{ZnDpa}$. The amine-presenting magnetic microspheres were treated with succinic anhydride, and the resulting carboxylic group was activated with $N$-hydroxysuccinimide, which was coupled in situ with the amine group of the binuclear $\mathrm{ZnDpa}$ complex to give $\mathrm{ZnMMs}$. The X-ray photoelectron spectroscopy (XPS) analysis of the resulting ZnMMs showed two peaks at 1022 and $1044 \mathrm{eV}$, corresponding to $\mathrm{Zn} 2 \mathrm{p}_{3 / 2}$ and $\mathrm{Zn} 2 \mathrm{p}_{1 / 2}$ (Fig. 2b). ${ }^{50,51}$ However, no apparent peak was observed in the same region for the bare magnetic microspheres (without the $\mathrm{Zn}^{2+}$ ion). This result clearly indicates the successful immobilization of the binuclear ZnDpa complex on the magnetic particle's surface.

Next, we performed the enrichment of phosphopeptides using $\beta$-casein, a standard protein with known phosphorylated sites, to assess the capability of the ZnMMs which can selectively enrich phosphopeptides. The MALDI-TOF MS spectra of the $\beta$-casein 

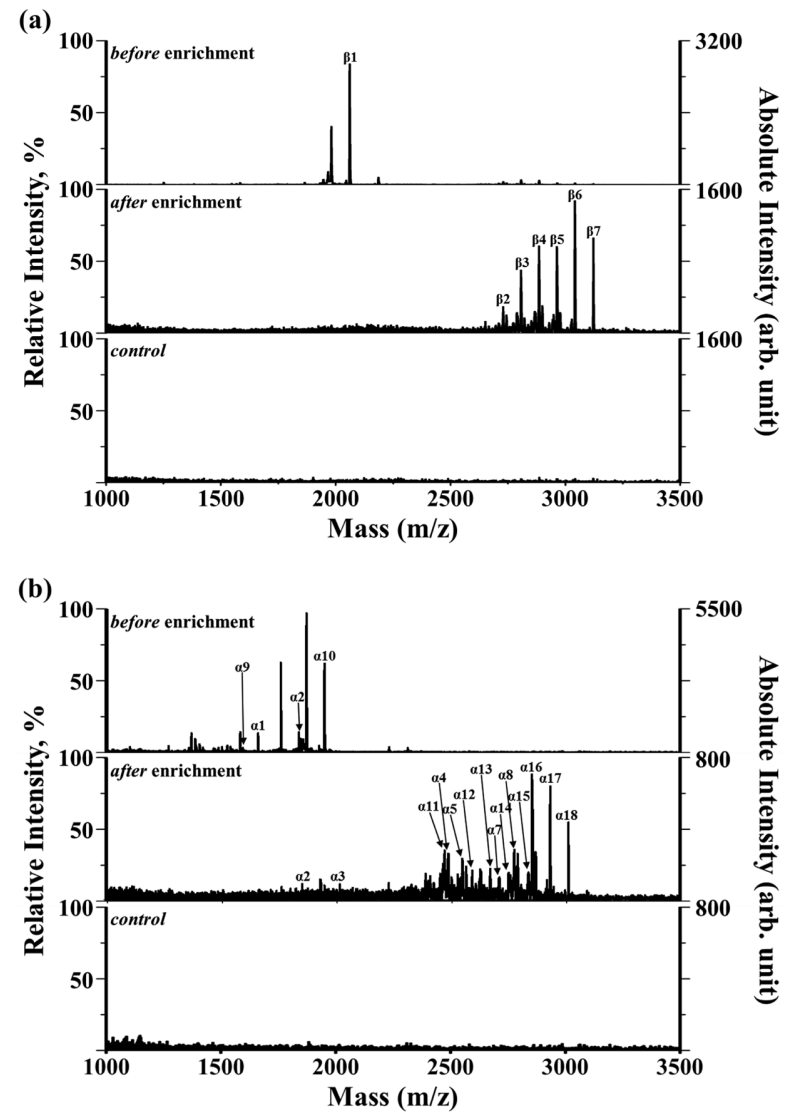

Fig. 3 The phosphopeptide enrichment from $\beta$-casein $(200 \mu \mathrm{M}$, $100 \mu \mathrm{L})$ (a) and $\alpha$-casein $(200 \mu \mathrm{M}, 100 \mu \mathrm{L})$ (b) using ZnMMs. The MALDI-TOF MS spectra of the casein digest before (top) and after enrichment (middle) revealed that the phosphorylated peptides were successfully enriched and could be analyzed with a clean background. As a control, the signal of phosphopeptides was not observed without the $\mathrm{Zn}^{2+}$ ion (bottom).

digest before and after enrichment are presented in Fig. 3a. Before the enrichment, several phosphopeptides were detected to have low intensities, except for a mono-phosphorylated peptide $\beta 1$ at $\mathrm{m} / z$ of 2060.2 . The multi-phosphorylated peptides had lower degree of intensity than that of mono-phosphorylated peptides due to poor ionization efficiencies in MALDI-TOF MS of multi-phosphorylated peptides. ${ }^{52}$ After enrichment by ZnMMs, however, six dominant peaks were distinctly identified with a clean background, corresponding to phosphorylated peptides $(\beta 2, \beta 3, \beta 4, \beta 5, \beta 6$, and $\beta 7$ at $\mathrm{m} / \mathrm{z}$ of $2725.8,2805.9$, $2884.6,2962.0,3040.7$, and 3120.8), while the major peaks of abundant non-phosphopeptides vanished. Additionally, multiphosphorylated peptides $(\beta 3, \beta 4, \beta 5, \beta 6$, and $\beta 7)$, including two-four phosphorylation sites were particularly observed, demonstrating that ZnMMs have a greater degree of affinity for multi-phosphorylated peptides compared to monophosphorylated peptides. The selective enrichment of the phosphopeptide from the $\beta$-casein digest was also examined using bare MMs (without the $\mathrm{Zn}^{2+}$ ion) as a control group. The phosphopeptide signal was barely detected in the spectrum. This result confirms that the binuclear ZnDpa complex played a key role in the enrichment of multi-phosphorylated peptides. Furthermore, phosphopeptide enrichment from the $\alpha$-casein tryptic digest, which consisted of two subunits ( $\alpha$-S1 and $\alpha-\mathrm{S} 2)$, was performed using ZnMMs (Fig. 3b). Without the pretreatment procedure, four mono-phosphorylated peptides $(\alpha 1$ and $\alpha 2$ at

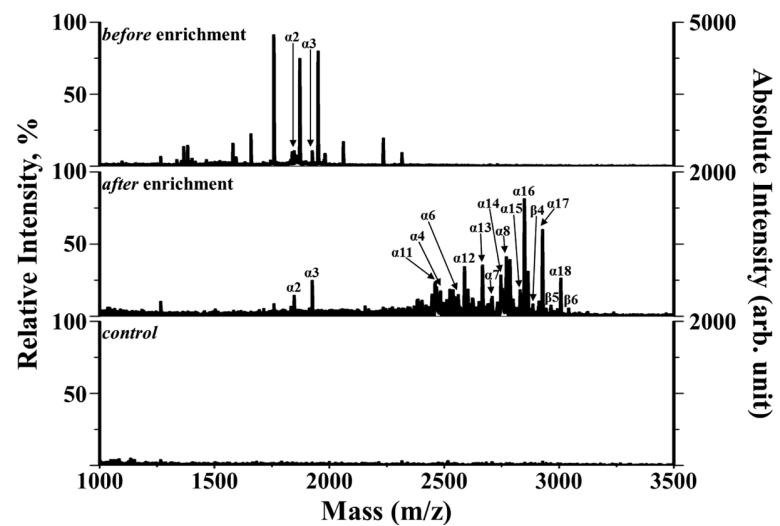

Fig. 4 The phosphopeptide enrichment from the mixed sample $(100 \mu \mathrm{L})$ of $\beta$-casein $(100 \mu \mathrm{M})$ and $\alpha$-casein $(50 \mu \mathrm{M})$ using ZnMMs. The MALDI-TOF MS spectra of the casein digest before (top) and after (middle) enrichment clearly illustrate the selective enrichment of multi-phosphopeptides $(\alpha 3, \alpha 4, \alpha 6, \alpha 7, \alpha 12, \alpha 13, \alpha 14, \alpha 15, \alpha 16$, $\alpha 17, \alpha 18, \beta 4, \beta 5, \beta 6)$ from the digest mixture of $\beta$-casein and $\alpha$-casein. As a control, when using bare magnetic microspheres (without a $\mathrm{Zn}^{2+}$ ion), the signals of phosphopeptides were not observed (bottom).

$\mathrm{m} / z$ of 1659.0 and 1847.3 from $\alpha$-S1, and $\alpha 9$ and $\alpha 10$ at $\mathrm{m} / z$ of 1592.8 and 1950.3 from $\alpha$-S2) were identified. However, after the enrichment with ZnMMs, 13 phosphopeptides $(\alpha 2, \alpha 3, \alpha 4$, $\alpha 5, \alpha 7, \alpha 8$, and $\alpha 9$ at $\mathrm{m} / z$ of $1847.3,1925.9,2482.2,2544.3$, 2666.8, 2703.6, and 2773.0 from $\alpha$-S 1 and $\alpha 12, \alpha 13, \alpha 14, \alpha 16$, $\alpha 17$, and $\alpha 18$ at $\mathrm{m} / z$ of $2461.7,2588.1,2746.7,2847.9,2927.9$, and 3007.9 from $\alpha$-S2) were observed as dominant peaks in the spectra and $69 \%$ of the enriched peptides were multiphosphorylated peptides. Similar to the results with $\beta$-casein, the enrichment of phosphopeptides from the $\alpha$-casein digest using bare MMs did not afford any phosphopeptide-related peaks.

The high selectivity of this enrichment towards multiphosphorylated peptides was further demonstrated using a tryptic digest of an $\alpha$-casein and $\beta$-casein mixture (Fig. 4). The mass spectra of the tryptic digest of the casein mixture prior to enrichment showed peaks mostly corresponding to nonphosphorylated peptides, except for two phosphopeptides ( $\alpha 2$ and $\alpha 3$ ) with low intensities. After the enrichment, 17 phosphopeptides from the $\alpha, \beta$-casein mixture were clearly observed and $82 \%$ (14 out of 17) of the enriched phosphopeptides were multi-phosphorylated peptides, indicating that ZnMMs have excellent enrichment efficiency and strong affinity towards multi-phosphorylated peptides. The detailed information for the phosphopeptides from the tryptic digest of $\alpha$-casein and $\beta$ casein are listed in Table $\mathrm{S} 1$ in Supporting Information. To date, only a few studies have reported the specific enrichment of multi-phosphorylated peptides. ${ }^{53-55}$ Our method, particularly, harnesses a designed artificial phosphate group receptor and uses magnetic particles, which avoids expensive reagents and elaborate procedures. Therefore, it can be an excellent alternative for the conventional enrichment of multi-phosphorylated peptides.

Since the phosphopeptide concentration in complex biological solutions is much lower, the ability to enrich multiphosphorylated peptides from a highly diluted solution is a key criterion to evaluate the efficiency of phosphopeptide enrichment. Thus, the detection sensitivity of the phosphopeptide enrichment using ZnMMs was investigated using a tryptic digest of $\beta$-casein at different concentrations ( $250 \mathrm{pmol}$ to $25 \mathrm{fmol}$ ). As shown in Fig. 5, the signal intensity of the phosphopeptides decreased as the concentration of $\beta$-casein decreased. The limit 


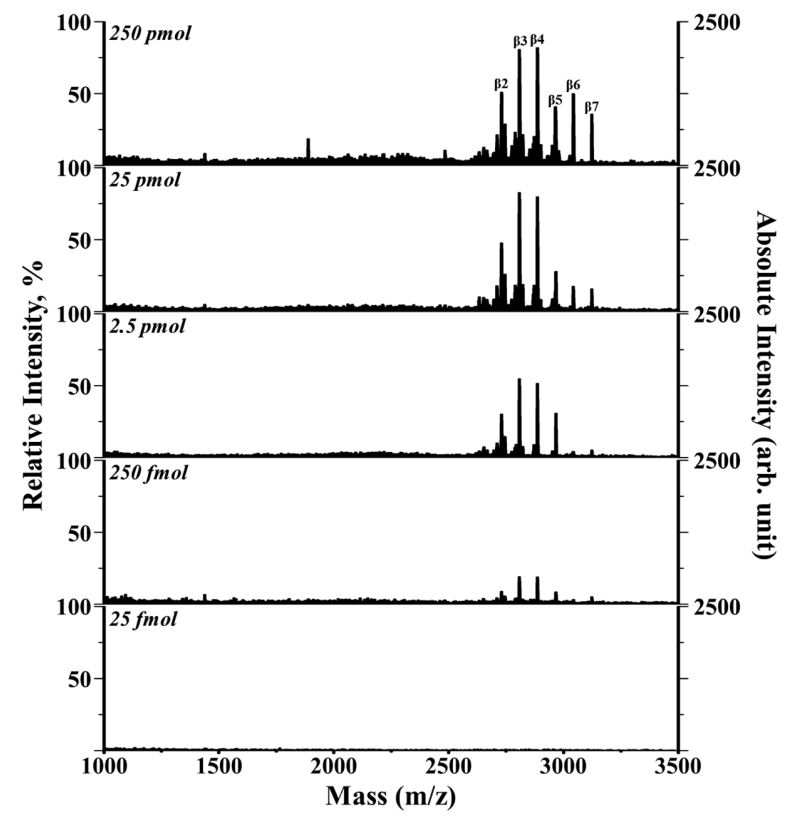

Fig. 5 The LOD of ZnMMs for phosphopeptides enrichment was investigated using tryptic digests of $\beta$-casein at different concentrations, ranging from $250 \mathrm{pmol}$ to $25 \mathrm{fmol}$. The LOD for $\beta$-casein was $250 \mathrm{fmol}$, with a good signal to noise ratio $(S / N>4.0)$.

of detection (LOD) for $\beta$-casein was $250 \mathrm{fmol}$ with a good signal to noise ratio $(S / N>4.0)$. These results led us to believe that ZnMMs can be used for the selective enrichment of multiphosphorylated peptides.

To further evaluate the ability of ZnMMs to enrich multiphosphorylated peptides in complex samples, ZnMMs were applied to mixtures of $\beta$-casein and BSA with different molar ratios. Before enrichment, only one phosphopeptide peak $(\beta 1)$ was detected at a molar ratio of 1:10 (Fig. 6(a)). By increasing the BSA content (molar ratio of 1:100), the $\beta 1$ peak disappeared, indicating that the low phosphopeptide levels were concealed by an increase in non-phosphopeptides in the mixture. After the enrichment, $7 \beta$-casein phosphopeptides were clearly observed with high responses and $71 \%$ of the enriched peptides were multi-phosphorylated peptides (Fig. 6(b)). To identify the detection capacity of ZnMMs, the intensity of the enriched phosphopeptides was also measured in comparison to the intensity of an internal standard peptide (ISP, $\mathrm{m} / \mathrm{z}$ of 1334). The ISP was added to the samples with precision at a known quantity to allow for the quantification of the mass spectrum directly. As shown in Fig. 6, the intensity of the ISP and the enriched phosphopeptides were almost the same in the mass spectrum. This result confirms that ZnMMs can enrich phosphopeptides in a mixture with a high $S / N$ ratio and a clean background without being hampered by highly abundant non-phosphorylated peptides.

\section{Conclusions}

In summary, the silica-based superparamagnetic microspheres were bound to the binuclear $\mathrm{ZnDpa}$ complex to yield $\mathrm{ZnMMs}$. The resulting ZnMMs were highly selective towards multiphosphorylated peptides and were easily separated from the solution using a magnet. ZnMMs exhibited high efficiency for the selective enrichment of trace amounts of phosphorylated peptides from tryptic digests of $\alpha$-casein and $\beta$-casein, and $82 \%$
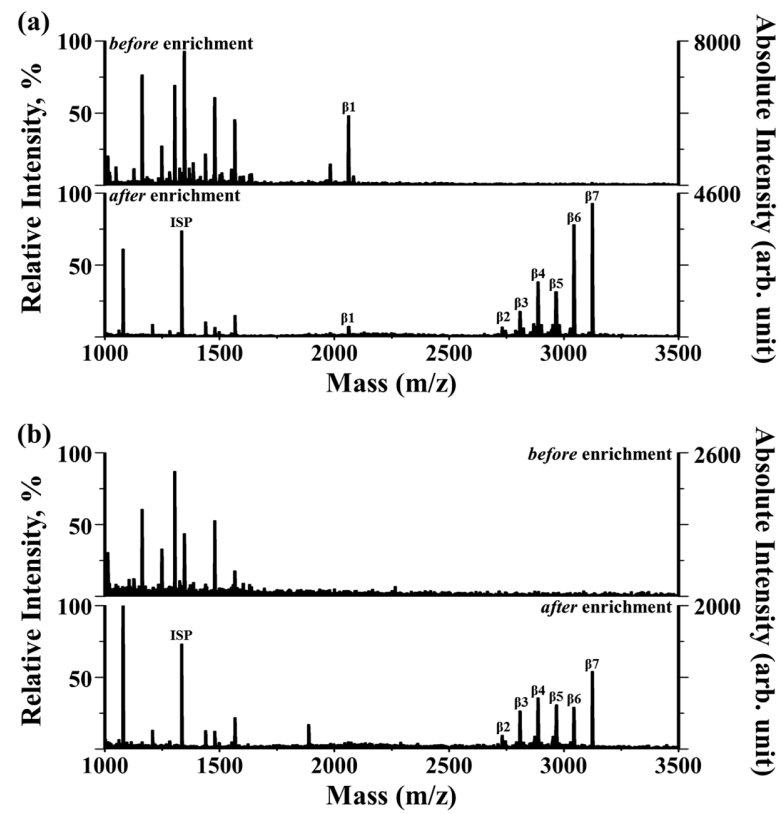

Fig. 6 The efficiency of ZnMMs for capturing phosphopeptides from a protein mixture. The MALDI-TOF MS spectra of the tryptic digest from a mixture $(100 \mu \mathrm{L})$ of $\beta$-casein $(1 \mu \mathrm{M})$ and BSA $(10 \mu \mathrm{M})$ (a) and $\beta$-casein $(1 \mu \mathrm{M})$ and BSA $(100 \mu \mathrm{M})(\mathrm{b})$. The internal standard peptide (ISP) with $\mathrm{m} / \mathrm{z}$ of 1334

of the enriched peptides were multi-phosphorylated peptides, due to the binuclear $\mathrm{ZnDpa}$ 's specific binding ability of phosphate groups. It is worth noting that the selective enrichment of multi-phosphorylated peptides from the mixture of $\beta$-casein and BSA with a molar ratio of 1:100 using ZnMMs was achieved. The MS intensity of the multi-phosphorylated fragments from $\beta$-casein exhibited very similar profiles in the $\beta$-casein and BSA mixtures with a molar ratio of $1: 10$ and $1: 100$, verifying the detection capacity of ZnMMs. Since the investigation of multi-phosphorylated peptide enrichment is significant for defining protein kinase substrates and revealing the activation states of signal transduction pathways, we believe that this technique can be further extended as a practical tool to a variety of biological samples for biomarker discovery.

\section{Acknowledgements}

This research was supported by the Priority Research Centers Program (2009-0093824) and Basic Science Research Program (NRF-2016R1D1A1A09918111) through the National Research Foundation (NRF) of Korea funded by the Ministry of Education and by the Agri-Bio Industry Technology Development Program (316028-3, Korea Institute of Planning and Evaluation for Technology in Food, Agriculture, Forestry and Fisheries (IPET)) and by the Big Issue Program funded by the KITECH, Republic of Korea (Project No. EO17470).

\section{Supporting Information}

Detailed synthetic procedure of materials associated with this article can be found in Supporting Information. This material is available free of charge on the Web at http://www.jsac.or.jp/ analsci/. 


\section{References}

1. S. Ivakhno and A. Kornelyuk, Biochemistry [Moscow], 2006, 71, 1060.

2. G. Manning, D. B. Whyte, R. Martinez, T. Hunter, and S. Sudarsanam, Science, 2002, 298, 1912.

3. J. V. Olsen, B. Blagoev, F. Gnad, B. Macek, C. Kumar, P. Mortensen, and M. Mann, Cell, 2006, 127, 635.

4. M. J. Hubbard and P. Cohen, Trends Biochem. Sci., 1993, $18,172$.

5. B. Salovska, A. Tichy, M. Rezacova, J. Vavrova, and E. Novotna, Rev. Anal. Chem., 2012, 31, 29.

6. X.-S. Li, G.-T. Zhu, Y.-B. Luo, B.-F. Yuan, and Y.-Q. Feng, TrAC, Trends Anal. Chem., 2013, 45, 233.

7. A. Leitner, TrAC, Trends Anal. Chem., 2010, 29, 177.

8. R. Abersold and M. Mann, Nature, 2003, 422, 198.

9. F. Wolschin, U. Lehmann, M. Glinski, and W. Weckwerth, Rapid Commun. Mass Spectrom., 2005, 19, 3626.

10. Y. D. Xu, M. L. Bruening, and J. T. Watson, Mass Spectrom. Rev., 2003, 22, 429.

11. B. Domon and R. Aebersold, Science, 2006, 312, 212.

12. S. P. Gygi, G. L. Corthals, Y. Zhang, Y. Rochon, and R. Aebersold, Proc. Natl. Acad. Sci. U. S. A., 2000, 97, 9390.

13. M. Schilling and D. R. Knapp, J. Proteome Res., 2008, 7, 4164.

14. A. Schmidt, E. Csaszar, G. Ammerer, and K. Mechtler, Proteomics, 2008, 8, 4577.

15. Y. Zhao, L. Wang, Z. Guo, X. Chi, X. Ma, Y. Qi, S. Fang, X. Li, and X. Liang, Chem. Res. Chin. Univ., 2015, 31, 44.

16. Y. Oda, T. Nagasu, and B. T. Chait, Nat. Biotechnol., 2001, $19,379$.

17. F. Zappacosta, G. F. Scott, M. J. Huddleston, and R. S. Annan, J. Proteome Res., 2015, 14, 997.

18. C.-F. Tsai, C.-C. Hsu, J.-N. Hung, Y.-T. Wang, W.-K. Choong, M.-Y. Zeng, D.-Y. Lin, R.-W. Hong, T.-Y. Sung, and Y.-J. Chen, Anal. Chem., 2014, 86, 685.

19. Z. Xiong, L. Zhang, C. Fang, Q. Zhang, Y. Ji, Z. Zhang, W. Zhang, and H. Zou, J. Mater. Chem. B, 2014, 2, 4473.

20. J. D. Dunn, J. T. Watson, and M. L. Bruening, Anal. Chem., 2006, 78, 1574.

21. C. E. Hydon, P. A. Eyers, L. D. Aveline-Wolf, K. A. Resing, J. L. Maller, and N. G. Ahn, Mol. Cell Proteomics, 2003 , 10,1055

22. X.-S. Li, X. Chen, B.-F. Yuan, and Y.-Q. Feng, RSC Adv., 2015, 5, 7832 .

23. I. Fukuda, Y. Hirabayashi-Ishioka, I. Sakikawa, T. Ota, M. Yokoyama, T. Uchiumi, and A. Morita, J. Proteome Res., 2013, 12, 5587.

24. H. Wang, J. Duan, and Q. Cheng, Anal. Chem., 2011, 83, 1624.

25. K. J. Shiau, S.-U. Hung, H.-W. Lee, and C.-C. Wu, Analyst, 2011, 136, 1922

26. Y. Yan, Z. Zheng, C. Deng, X. Zhang, and P. Yang, Chem. Commun., 2013, 49, 5055

27. H. J. Zhou, R. J. Tain, M. L. Ye, S. Y. Xu, S. Feng, C. S. Pan, X. G. Jiang, X. Li, and H. F. Zou, Electrophoresis, 2007, 28, 2201.
28. S.-S. Liang, H. Makamba, S.-Y. Huang, and S.-H. Chen, J. Chromatogr. A, 2006, 1116, 38.

29. J. T. Wilson-Grady, J. Villen, and S. P. Gygi, J. Proteome Res., 2008, 7, 1088.

30. A. B. Iliuk, V. A. Martin, B. M. Alicie, R. L. Geahlen, and W. A. Tao, Mol. Cell. Proteomics, 2010, 9, 2162.

31. J.-M. Lehn, Angew. Chem., Int. Ed., 1998, 27, 89.

32. S. K. Kim, D. H. Lee, J.-I. Hong, and J. Yoon, Acc. Chem. Res., 2009, 42, 23.

33. A. Ojida, Y. Mito-oka, M. Inoue, and I. Hamachi, J. Am. Chem. Soc., 2002, 124, 6256

34. A. Ojida, Y. Mito-oka, M. Inoue, and I. Hamachi, J. Am. Chem. Soc., 2003, 125, 10184

35. A. Ojida, Y. Mito-oka, K. Sada, and I. Hamachi, J. Am Chem. Soc., 2004, 126, 2454.

36. A. Ojida, M. Inoue, Y. Mito-oka, H. Tsutsumi, K. Sada, and I. Hamachi, J. Am. Chem. Soc., 2006, 128, 2052.

37. A. Ojida, T. Sakamoto, M. Inoue, S.-H. Fujishima, G. Lippens, and I. Hamachi, J. Am. Chem. Soc., 2009, 131, 6543.

38. T. Sakamoto, A. Ojida, and I. Hamachi, Chem. Commun., 2009, 45, 141.

39. D. Kraskouskaya, J. A. Drewry, E. Duodu, S. Burger, J. Eaton, G. A. Cisneros, and P. T. Gunning, Med. Chem. Commun., 2013, 4, 289.

40. J. H. Kang, H. J. Kim, T.-H. Kwon, and J.-I. Hong, J. Org Chem., 2014, 79, 6000.

41. S. Zhang, L. Han, C.-G. Li, J. Wang, W. Wang, Z. Yuan, and X. Gao, Tetrahedron, 2012, 68, 2357.

42. J. Gao, H. Gu, and B. Xu, Acc. Chem. Res., 2009, 42, 1097.

43. W.-F. Ma, Y. Zhang, L.-L. Li, L.-J. You, P. Zhang, Y.-T. Zhang, J.-M. Li, M. Yu, J. Guo, H.-J. Lu, and C.-C. Wang, ACS Nano, 2012, 6, 3179.

44. F. Xu, J. H. Geiger, G. L. Baker, and M. L. Bruening, Langmuir, 2011, 27, 3106.

45. H. Chen, C. Deng, and X. Zhang, Angew. Chem., Int. Ed., 2010, 49, 607.

46. S. Liu, H. Chen, X. Lu, C. Deng, X. Zhang, and P. Yang, Angew. Chem., Int. Ed., 2010, 49, 7557.

47. Bioclone Inc. Homepage, http://www.bioclone.us/

48. Preliminary data of this work was presented at the International Conference. S. W. Bae, S. Kim, S. H. Shin, and D. Lee, in Proceedings of 2016 IEEE SENSORS, 2016, Orlando, FL, 1.

49. C. Lakshimi, R. G. Hanshaw, and B. D. Smith, Tetrahedron, 2004, 60, 11307.

50. M. C. Biesinger, L. W. M. Lau, A. R. Gerson, and R. St.C. Smart, Appl. Surf. Sci., 2010, 257, 887.

51. J. Chen, S. Shen, P. Guo, P. Wu, and L. Guo, J. Mater Chem. A, 2014, 2, 4605.

52. J. N. M. Ballard, G. A. Lajoie, and K. K. C. Yeung, J. Chromatogr. A, 2007, 1156, 101.

53. H. Zhong, X. Xiao, S. Zheng, W. Zhang, M. Ding, H. Jiang, L. Huang, and J. Kang, Nat. Commun., 2013, 4, 1656.

54. L.-P. Li, T. Zheng, L.-N. Xu, Z. Li, L.-D. Sun, Z.-X. Nie, Y. Bai, and H.-W. Liu, Chem. Commun., 2013, 49, 1762.

55. X. Li, Z. Guo, Q. Sheng, X. Xue, and X. Liang, Analyst, 2012, 137, 2774 